\title{
Radiated Suceptibility Investigation of Electronic Board from Near Field Scan Method
}

\author{
Nicolas Lacrampe, Sebastien Serpaud, \\ EMC design support unit, NEXIO, \\ Toulouse, France, \\ sebastien.serpaud@nexio.fr, \\ nicolas.lacrampe@nexio.fr
}

\author{
Alexandre Boyer \\ CNRS, LAAS, 7 avenue du colonel \\ Roche \\ F-31400 Toulouse, France, \\ Université de Toulouse, INSA, LAAS \\ F-31400 Toulouse, France \\ alexandre.boyer@laas.fr
}

\author{
Séreirath Tran \\ Airbus Operations SAS \\ Toulouse, France \\ sereirath.tran@airbus.com
}

\begin{abstract}
This paper aims at studying the near field measurement method in immunity to investigate the robustness of components and electronic boards. An ARINC receiver is used as case study. The experimental set-up, the probe calibration method and the experimental results are presented. Investigation method and results are discussed and compared to normative measurement method like TEM cell measurement.
\end{abstract}

Keywords - investigation; robustness; radiated immunity, near field; Component; electronic board.

\section{INTRODUCTION}

The immunity management of electronic boards is a hard challenge to maintain competitiveness of product. The development time is more and more reduced by market requirements and normative constraints become stronger. Moreover, the complexity of electronic board is increasing generation after generation, as the immunity to electromagnetic disturbance issue.

Current EMC test methods do not always allow a fast search of the root-causes of non-compliance to immunity requirements. Radiated immunity tests in anechoic chamber allow the measurement of robustness levels of equipment. Unfortunately this method requires very powerful amplifiers to provide the required electric field levels. The test in Mode Stirred Reverberation Chamber (MSRC) provides higher levels but it fails, as the anechoic chamber method, to identify the actual source of non-compliance. These last ten years, several preliminary academic works have been done to investigate the susceptibility of integrated circuits. The injection method was able to trigger failures in digital circuits [1] [2], microcontrollers [3] [4], analog-to-digital converter [5], operational amplifiers [6], oscillators and PLL [3] [6] [7].

This paper presents a study based on near field measurement to investigate the immunity issues of electronic boards. The objective of this study is to evaluate the ability of this measurement method to quickly identify weak design which reduces the robustness of electronic boards. The near field measurement in immunity proposes the construction of a map of the susceptibility of a device under test. The localization of weak design is the first step to improve electronic boards or components robustness and build up EMC guidelines.

This work was supported by EFT-SAFE3A (Electrical Fast Transient SAFE Embedded electronics for Aeronautic and Automotive Applications) research project. (Project reference: ANR-14-CE22-0022)
The following section will depict the test bench and calibration method used to near field immunity investigation. In section III, we will present device used as study case. Internal structure for device and Pass/Fail criteria are described. In section IV, we will describe and discuss about the results of the near field immunity measurement. To conclude, the near field measurement results are compared to the measurement from TEM cell method in section V.

\section{THE NEAR FIELD MEASUREMENT IN IMMUNITY}

The aim of the near-field scan immunity (NFSi) method is to produce a high level of disturbance above device under test (DUT). A miniature near-field injection probe is used to produce a strong localized field near the DUT. This method is very suitable to characterize robustness level of electronic board or circuit. The displacement of the injection probe above the DUT ensures the construction of a susceptibility map and the localization of design issues, such as external disturbance coupling area or sensitive circuit pins.

\section{A. Test bench presentation}

The complete set-up is described in the next figure. The immunity test is done in frequency domain. The disturbance is produced by a Continuous Wave $(\mathrm{CW})$ generator followed by a 10 - $150 \mathrm{~W}$ power amplifier. The RF signal could be modulated, for example by a low frequency square signal, as suggested by DO160 standard.

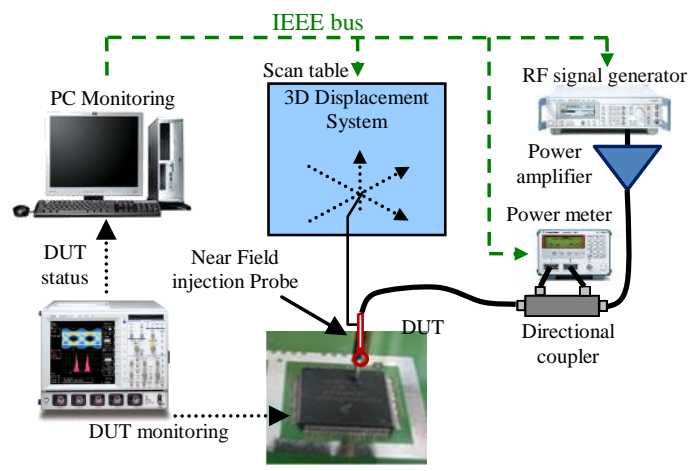

Fig 1. Test bench description

A bidirectional coupler is used to measure the amount of forward and reflected power. This information is used to 
evaluate the voltage induced on the device under test from the calibration of the probe and the losses of the different equipments of the injection system (power amplifier, coupler, cables and injection probe). All the equipments are monitored by BAT-EMC software to automate test and generate result reports.

\section{B. Near field probe \& Calibration}

Near-field probes are miniatures and wideband antennas usually dedicated to sense either electric or magnetic fields near to electronic components, printed circuit boards or antennas. They are usually based on small loops and opened tips. When they are excited by an electrical signal, they can produce a strong electric or magnetic fields in their near-field region, which can couple on a DUT to produce important voltage fluctuations locally on a track or on a package pin.

In this study, handmade tangential magnetic field probes are considered. They are designed with RG405 semi-rigid coaxial cable. Two magnetic field probes have been designed and tested. The probe which produces the strongest magnetic field will be selected for the susceptibility tests. Their structures and their dimensions are shown in Fig 2. They consist in 8 turn coils with a diameter equal to $5 \mathrm{~mm}$. The numbers of turns coils and the diameter of probe were chosen to have a good compromise between the spatial resolution of the probe and its coupling coefficient. A ferrite core has been introduced inside the coil of probe NFSi_8t-V1 in order to increase the magnetic flux inside and in the vicinity of the core.

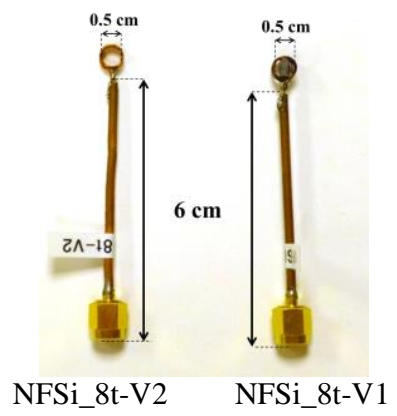

Fig 2. Dimensions of the tested near-field injection probe

Fig 3. compares both probe impedance results between measurements and 3D simulations. For measurement, a simple S11 parameter with a network analyzer has been realized. For simulation, commercial electromagnetic software solutions [8] [9] were used in including all parts of probes (coaxial cables, wires and ferrite). Model of SMA connectors has not been used as negligible. The interest of these simulations is to design various probes in order to fit impedance and optimize probe size by changing different parameters like numbers of turns, probe diameter, wire size... Moreover, these simulations provide amplitude of the fields produced in the vicinity of the probe. For example, at $1 \mathrm{~mm}$ below the magnetic field probe NFSi_8t-V2, the magnetic field is about $180 \mathrm{~A} / \mathrm{m}$ at $100 \mathrm{MHz}$ when it is excited by a $85 \mathrm{~W}$ RF generator.

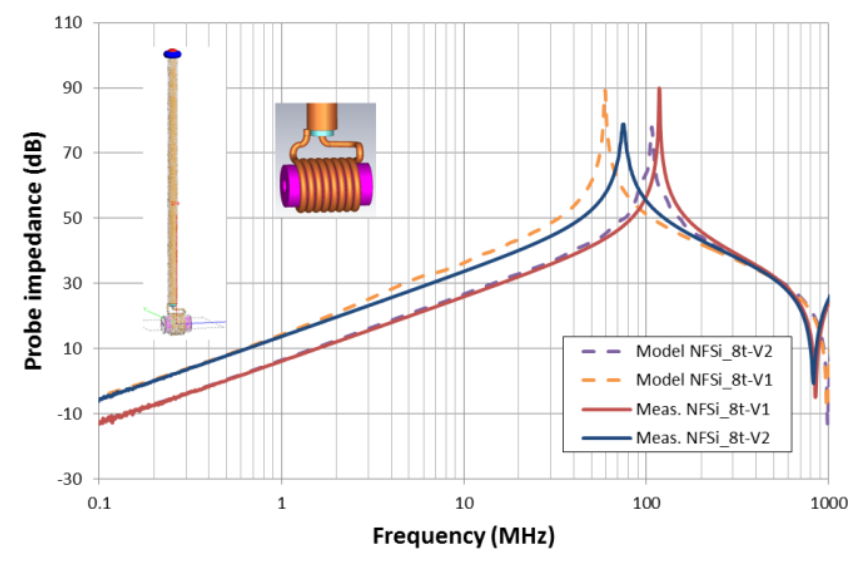

Fig 3. Comparison between measured and simulated probe impedance of 8 turn near-field injection probe (with and without ferrite)

The probe impedance measurements show a resonance frequency between $70 \mathrm{MHz}$ and $120 \mathrm{MHz}$, if the ferrite is inserted or not. However, this variation of probe impedance is compensated by the calibration method described below. A close loop regulation is used during the calibration to inject the same required level on all frequency range, regardless the probe chosen.

The spatial resolution of both probes is evaluated to $2.5 \mathrm{~mm}$ for a scan height equal to $1 \mathrm{~mm}$. Fig. 4 shows the voltage coupled on a micro-strip line for various position of the nearfield probe. The coil axis is perpendicular to the line to ensure an optimal coupling of the magnetic field to the line. At $1 \mathrm{~mm}$ above the line, a variation of $10 \mathrm{~dB}$ of the coupled voltage is measured between a point right above the line and a point by $2.5 \mathrm{~mm}$.
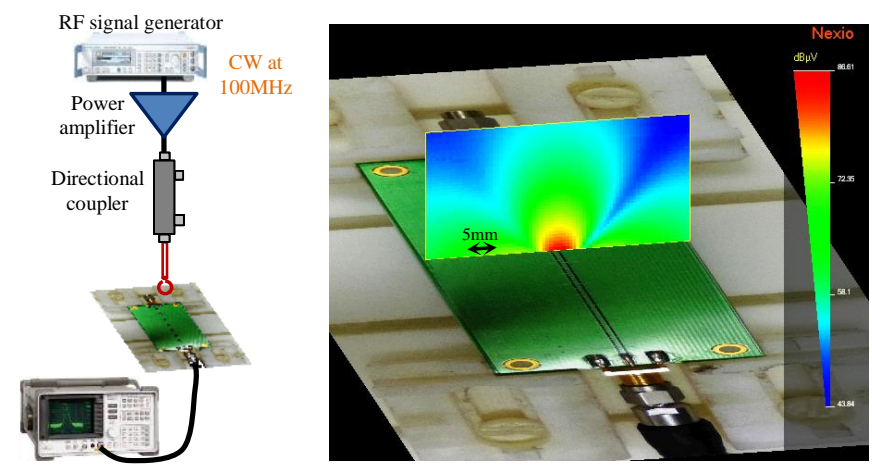

Fig 4. Resolution of near-field injection probe

The calibration of probe is an important consideration to quantify the measured susceptibility. Several parameters could be proposed to express the susceptibility level: the amplitude of the forward wave delivered to the probe, the excitation current, the magnetic field produced at a given distance... However, they do not give a direct indication about the effect on the DUT. We propose to calibrate the probes by the measurement of the required excitation amplitude to induce a given voltage on a reference device. The calibration coefficient is the ratio between the voltage induced on the reference device and the excitation amplitude (e.g. the forward power delivered to the 
probe). It is valid only for a given probe and a scan altitude. During a near-field injection test, if the DUT fails for a given forward power, the susceptibility level can be expressed as the voltage that the probe would induce on the reference device. Fig 5. describes the calibration experimental set-up. The reference device is a $4 \mathrm{~cm}$ round wire over a ground plane. The line is $50 \Omega$ adapted and terminated by $50 \Omega$ SMA loads. The injection probe is placed at $1 \mathrm{~mm}$ above the line and is exciting by a harmonic signal. The amplitude of the excitation is set to generate a constant voltage at the line terminal.
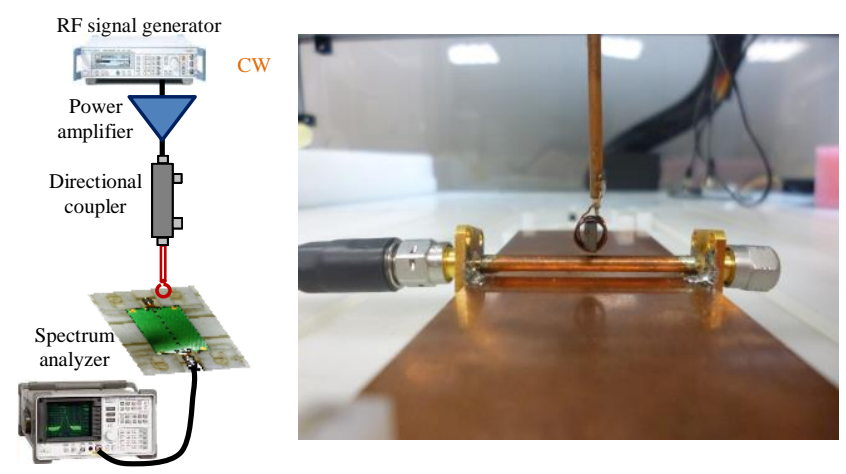

Fig 5. Probe calibration set-up

Fig 6. presents the calibration results of both magnetic field probes from $1 \mathrm{MHz}$ to $1 \mathrm{GHz}$, because the component tested in this study fails over this frequency range. The calibration shows that the introduction of ferrite increases by nearly $10 \mathrm{~dB}$ the injection level up to several $\mathrm{MHz}$ because it increases the magnetic flux. However, the ferrite also increases the probe inductance and limits the probe excitation current above $10 \mathrm{MHz}$. Above $100 \mathrm{MHz}$, the ferrite brings no advantages. The probe NFSi_8t-V1 is selected for the following susceptibility tests. In order to verify the relevance of the calibration results, simulations of the interaction between both probes and the reference line is developed. The magnetic-field probes are considered as inductors and the near-field radiation is computed using elementary magnetic dipole model. The voltages on the line terminal can be deduced by using the field coupling to transmission line equations. Fig 6. shows that the simulated calibration coefficients fit with the simulated one at least up to $300 \mathrm{MHz}$.

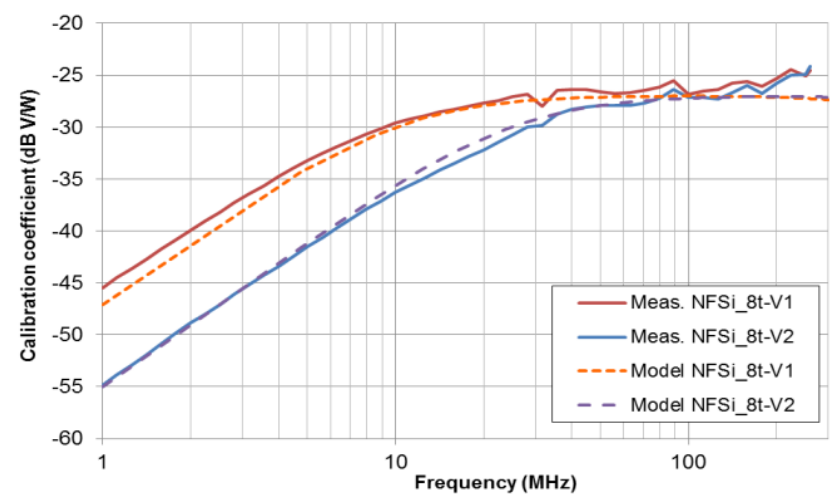

Fig 6. Comparison between measured and simulated calibration coefficients of both near-field injection probe

\section{DUT PRESENTATION}

The component used on our mockup is an ASIC (Application Specific Integrated Circuit) designed for AIRBUS company. This component is an eight channel line receiver for ARINC (Aeronautical Radio, Incorporated) 429 data transmission systems, as shown in the block diagram in Fig 7.

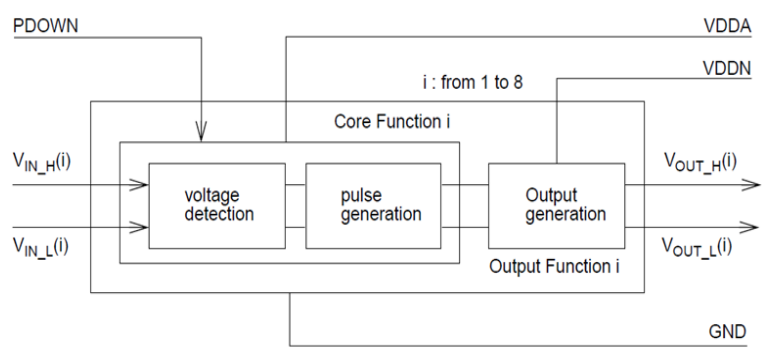

Fig 7. DEM8 ASIC block diagram

The ARINC 429 line receiver converts ARINC 429 differential RZ data to TTL/CMOS compatible differential outputs. The waveform of the ARINC 429 signals follows a differential bipolar RZ type. As shown in the transfer function diagram below in Fig 8., logic levels are determined by the voltage difference between the two communication lines.

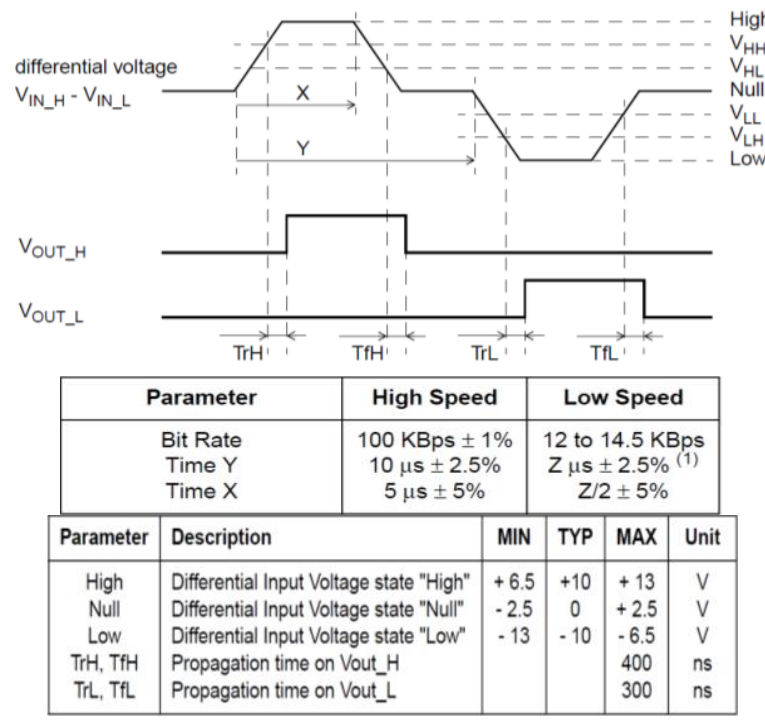

Fig 8. Transfer function diagram

The transmission orders are generated by an external signal generator and encoded in ARINC format by an ARINC driver. Encoding data are transmitted through a twisted pair cable to the ARINC receiver. Monitoring is carried out after decoding data phase (ARINC component output pins) as shown in Fig 9.

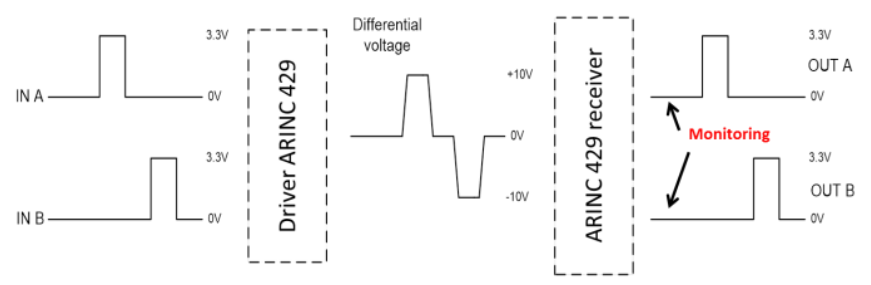

Fig 9. Encoding and decoding principle 
Pass/Fail criteria are given by the mask as shown in Fig 10. Indeed, the receiver ARINC is susceptible when the signal is out of the mask.

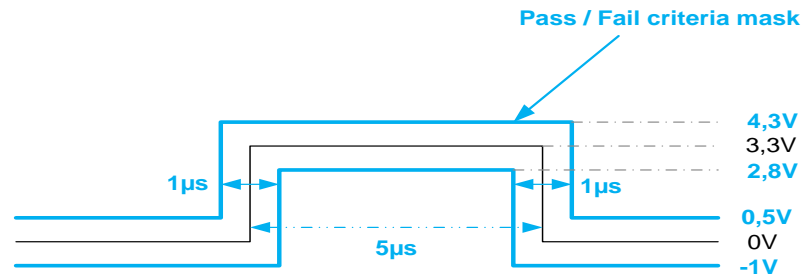

Fig 10. Pass/Fail criteria mask

Pinout of the ARINC components (TQFP44 package) are presented in Fig 11. Some pins, that were important in scan results analysis, are located.

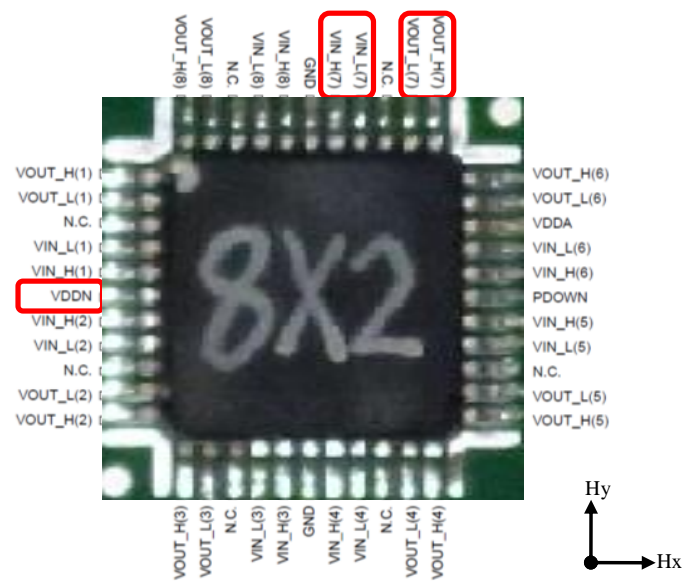

Fig 11. Pinout of ARINC receiver

\section{NFSI TEST RESUTS}

This chapter presents the near field immunity test results obtained on ARINC device. Tests were done following test bench description shown in Fig 1. with the probe NFSi_8t-V1. For this study, the input signal from the RF generator is CW signal. The RF signal frequency is swept from $1 \mathrm{MHz}$ to $1 \mathrm{GHz}$ $(1 \mathrm{MHz}$ linear step on frequency range $1 \mathrm{MHz}-10 \mathrm{MHz}$, $20 \mathrm{MHz}$ linear step on frequency range $20 \mathrm{MHz}-240 \mathrm{MHz}$, and $100 \mathrm{MHz}$ linear step on frequency range $300 \mathrm{MHz}-1 \mathrm{GHz}$ ). The frequencies ranges were cut like that to optimize the time of test and focus on critical frequencies. The scans are done twice, for two probe orientations ( $\mathrm{Hx}$ and $\mathrm{Hy}$ ).

ARINC device is monitoring by oscilloscope to control that the output signal is conformed to the selected criteria. Some defaults are observed: jitter on signal edges, modulation on high state of the ARINC signal, and no signal on input differential signals. A test algorithm is defined to check each point at the selected discrete frequency range. For each frequency, a progressive power is applied above DUT to target level. At each step, the device operation is controlled. If a fault is detected, an algorithm research is launched to detect the minimum level of disturbance to trigger the default.

As this operation is repeated for each point, a map of device susceptibility is built. The value displayed on each point is the minimum level of disturbance to create fault operation for each point at the selected frequency. The maximum power is limited by power amplifier. So, the red color indicates either a high immunity level of the DUT or a limitation of the injection system. In contrary, a blue color defined a low immunity level of the DUT (minimum level of disturbance to induce a failure).

The DUT presents different failures in frequency range $1 \mathrm{MHz}$ to $1 \mathrm{GHz}$. Fig 12. and Fig 13. show the near-field scan results for both orientations between 1 and $10 \mathrm{MHz}$. On both figures, the evolution of immunity level with the frequency, the susceptibility map and the localization of sensitive area are presented. Fig 14. , Fig 15. and Fig 16. present respectively the susceptibility map of the component at $100 \mathrm{MHz}, 200 \mathrm{MHz}$ and $800 \mathrm{MHz}$.
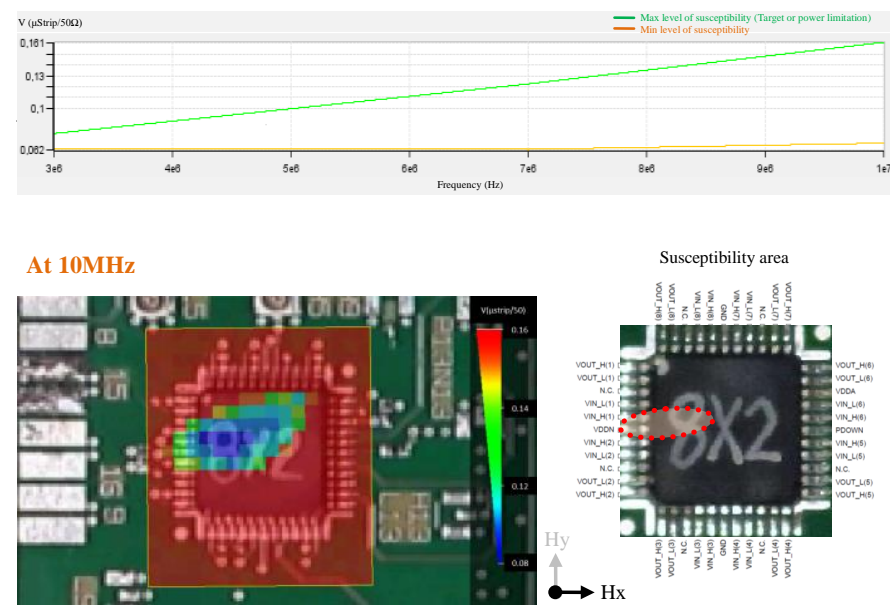

Fig 12. Hx Near-field immnity level (3MHz-10MHz)
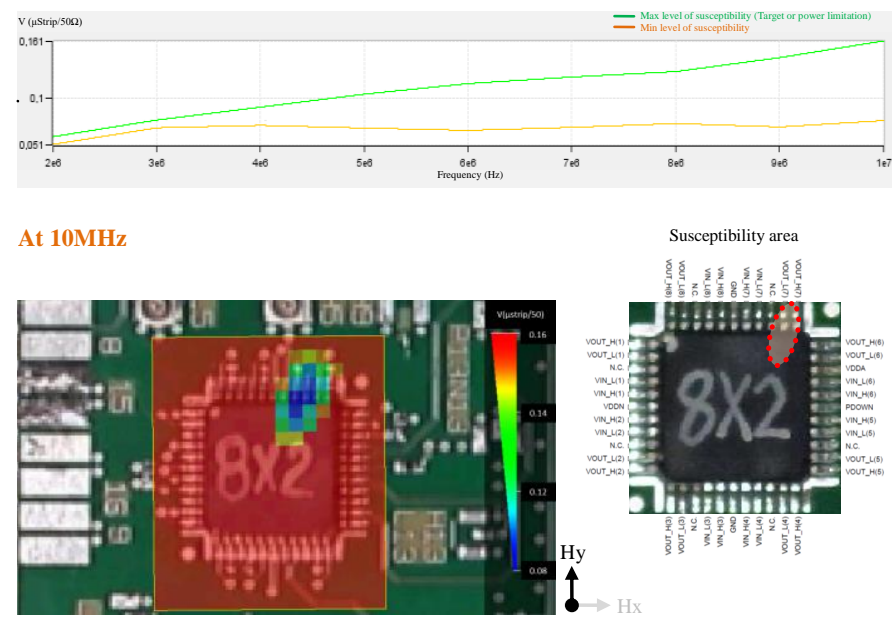

Fig 13. Hy Near-field immnity level (2MHz-10MHz) 


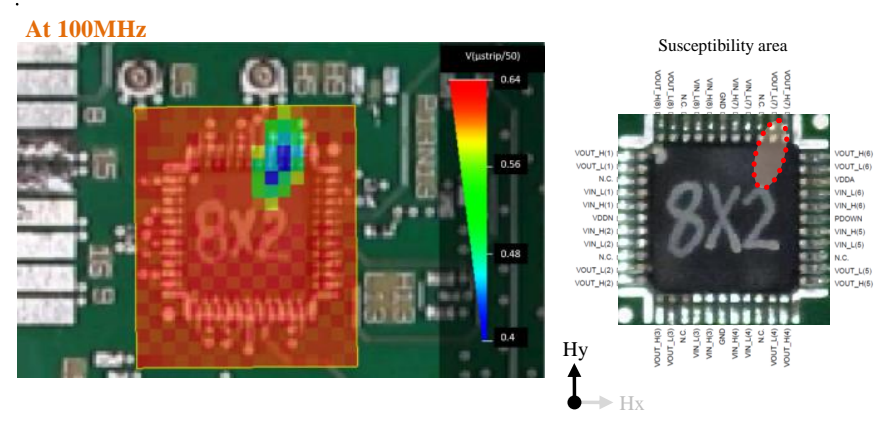

Fig 14. Hy Near-field immnity level at $100 \mathrm{MHz}$

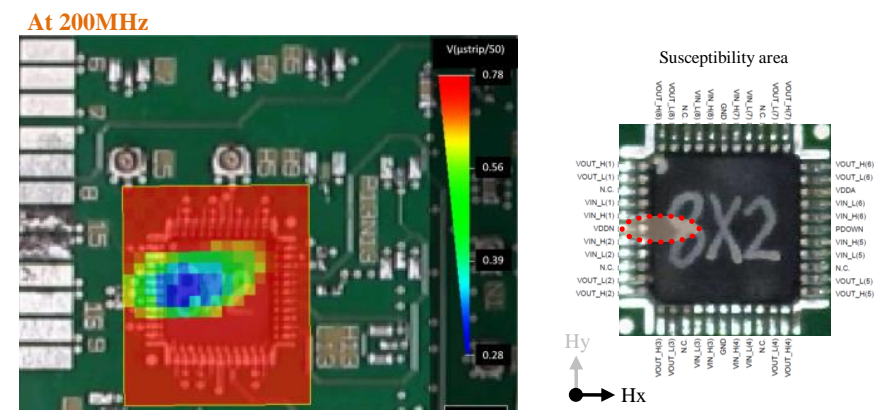

Fig 15. Hx Near-field immnity level at $200 \mathrm{MHz}$
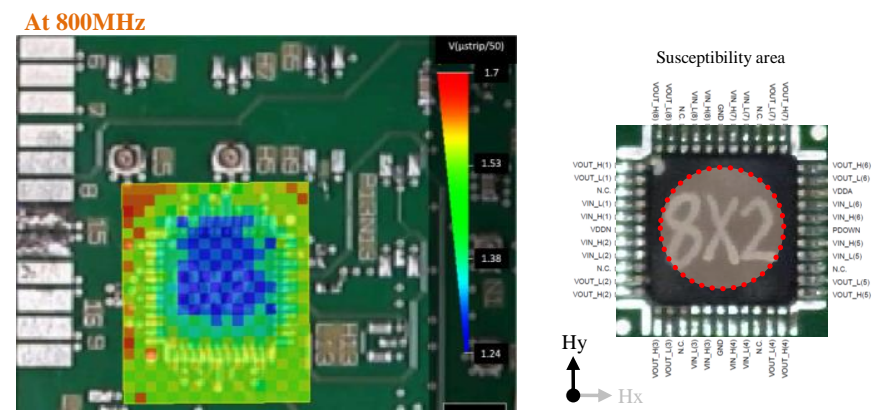

Fig 16. Hy Near-field immnity level at $800 \mathrm{MHz}$

The previous figures show the susceptibility of component and the localization of sensitive area are not the same following the frequency of disturbance. At low frequency and until $300 \mathrm{MHz}$, the susceptibility area is above the differential output and above VDD connection. At $800 \mathrm{MHz}$ the susceptibility area is above ARINC chip. When the disturbance is above differential output or VDD connection, the default is a modulation of disturbance on high state of output ARINC signal. Around $500 \mathrm{MHz}$, the default is a jitter on output signal if the disturbance is above differential output. And at $800 \mathrm{MHz}$, ARINC component is stopped in injecting a disturbance above the chip (no signal on differential output).

The near field scan method in immunity mode allows identifying susceptible pins easily and analyzing the observed failures. That wouldn't be possible with other radiated immunity methods using TEM cell, anechoic or reverberant chamber...

\section{CORRELATION WITH TEM CELL MEASUREMENT}

This aim of this chapter is to compare near field scan test in immunity with TEM cell test result. For this test, the same injection test bench used during NFSi test is taken to perturb ARINC component. The FCC-TEM-JM1 Fischer loaded with $50 \mathrm{ohms} / 100 \mathrm{~W}$ is used. To not damage the load, the power injected in TEM-cell was limited to $85 \mathrm{~W}$.

To determine the field strength equivalent in TEM-cell in first approximation, (1) can be used:

$$
P=\frac{(E * d)^{2}}{Z}
$$

$$
\begin{aligned}
& \mathrm{P}: \text { net power }(\mathrm{W}) \\
& \text { E: Field strength }(\mathrm{V} / \mathrm{m}) \\
& \mathrm{d}: \text { Separation floor-septum }(\mathrm{m}) \\
& \mathrm{Z} \text { : TEM characteristic impedance }(\Omega)
\end{aligned}
$$

Only for free space, an expression of the relationship between the magnetic field $\mathrm{H}$ and the electric field $\mathrm{E}$ (2) is the following:

$$
H=\frac{E}{Z_{0}}
$$

$\mathrm{H}$ : amplitude of magnetic field $(\mathrm{A} / \mathrm{m})$

E: amplitude of electric field (V/m)

$\mathrm{Z}_{0}$ : characteristic impedance of free space $=377 \Omega$

With TEM cell characteristic and a net power of $85 \mathrm{~W}$, the electric and magnetic field strength given by calculation above are around $1500 \mathrm{~V} / \mathrm{m}$ and $4 \mathrm{~A} / \mathrm{m}$ respectively. As mentioned in part II.B, the magnetic field within the TEM cell is nearly 45 times lower than that produced at $1 \mathrm{~mm}$ of the probe NFSi_8t$\mathrm{V} 2$ excited with the same net power.

To perturb only the ARINC component, all electrical board is covered by copper tape as shown on figure hereafter.

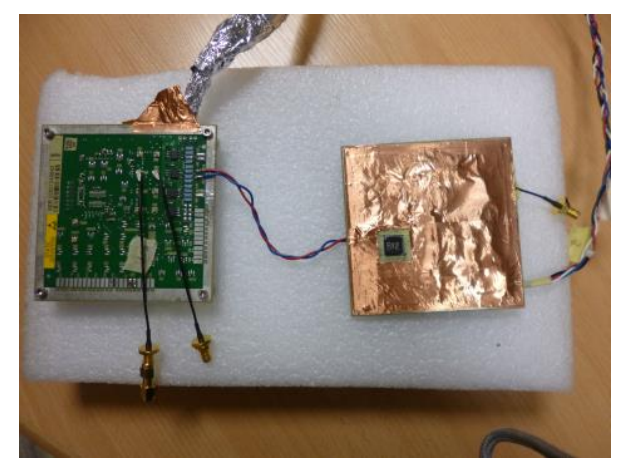

Fig 17. ARINC board modified for TEM measurement

Tests have been done from $1 \mathrm{MHz}$ to $1 \mathrm{GHz}(2 \mathrm{MHz}$ linear step on frequency range $1 \mathrm{MHz}-10 \mathrm{MHz}$, and $20 \mathrm{MHz}$ linear step on frequency range $20 \mathrm{MHz}-1 \mathrm{GHz}$ ). Both orientations of 
board $\left(0^{\circ}\right.$ and $\left.90^{\circ}\right)$ are done. During this test, the same monitoring that NFSi test is used. (Using an oscilloscope to control that the output signal conforms to the selected criteria defined in Fig 10. )

With this standard TEM test, the ARINC component presents no default in frequency range $1 \mathrm{MHz}$ to $1 \mathrm{GHz}$ in injecting $85 \mathrm{~W}$ at TEM cell input. Same test has been performed in removing the copper tape and no default was appeared. In order to understand the results obtained with both method (TEM and NFSi), a calibration test was performed by using a micro-strip loaded by a $50 \mathrm{ohms} \mathrm{SMA} \mathrm{on} \mathrm{one} \mathrm{side,} \mathrm{and}$ a spectrum analyzer on the other side. This micro-strip is a simple wire over a ground plane, placed on a $10 \mathrm{~cm} * 10 \mathrm{~cm}$ aluminum board. The distance between wire and ground plane is $350 \mu \mathrm{m}$ to have $50 \Omega$ adapted line. The wire length is around $7 \mathrm{~mm}$ to be equivalent to bonding connection.

During this calibration test, a CW sinusoidal excitation with a $37 \mathrm{~W}$ constant power was used on frequency range $1 \mathrm{MHz}$ to $1 \mathrm{GHz}$. According to TEM cell manufacturer, $37 \mathrm{~W}$ input power permits to achieve $1000 \mathrm{~V} / \mathrm{m}$ electric field. The TEM calibration test has consisted to inject excitation at TEM cell input and record the voltage measured at spectrum analyzer input. The NFSi calibration test was performed like shown on Fig 5. in limiting the power forward at $37 \mathrm{~W}$ to inject the same excitation in NFSi probe. The probe is placed at $1 \mathrm{~mm}$ above the line. To compare both injection methods, Fig 18. presents the coefficient calibration of each method.

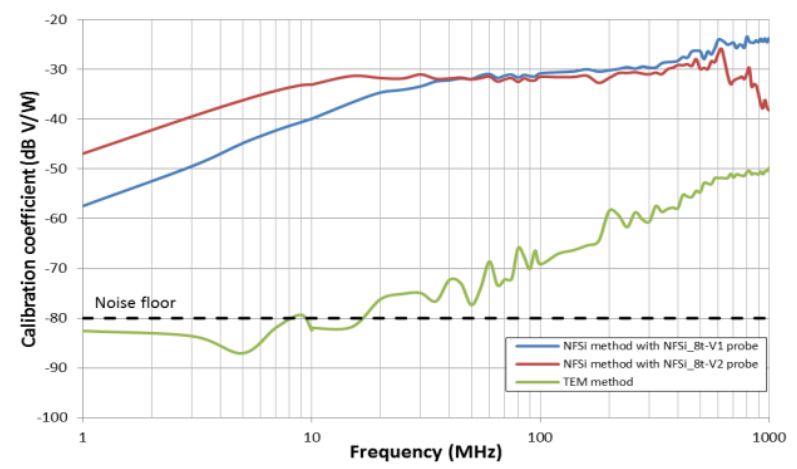

Fig 18. Comparison between TEM cell and NFSi calibration coefficients

These results show that it's necessary to have more power with TEM method than NFSi method to inject similar voltage induced on the wire. The distance between the EUT and the injection point (septum for TEM cell, and probe for NFSi) is different for each method. The greater this distance, the higher the power to be injected. So, to create a default on ARINC component with TEM method, the power injected at the TELL cell input should be around $20-30 \mathrm{~dB}$ more than power injected in the NFSi probe.

\section{CONCLUSION}

The results obtained on this ARINC component suggest the near field scan method in immunity is a relevant test method to localized susceptibility area on design. Precise analysis on failure can be done on the system to improve the design with direct local modifications. In this paper, NFSi results have been compared to results obtained with standard immunity method IEC 62132-2 using TEM cell. No default appeared on ARINC component with TEM cell method. The RF disturbance applied at the input to the TEM cell is related to the electromagnetic field by the distance between the septum and EUT. So, to inject similar disturbance when the NFSi probe is placed at $1 \mathrm{~mm}$ above EUT, the power applied at the TEM input should be at least 100 times more than the power applied on the NFSi probe. The advantage of NFSi method compared to TEM cell method is that no specific electrical board is necessary.

Other works are in progress to develop new algorithm to optimize the test duration and monitoring control of NFSi test bench.

\section{ACKNOWLEDGMENT}

This work benefited from the support of the project EFTSAFE3A (Electrical Fast Transient - SAFE Embedded electronics for Aeronautic and Automotive Applications) ANR-14-CE22-0022 of the French National Research Agency (ANR).

\section{REFERENCES}

[1] A. Boyer, E. Sicard, S. Bendhia, « Characterization of the Electromagnetic Susceptibility of Integrated Circuits using a Near Field Scan », Electronic Letters, 4th January 2007, vol. 43, No 1.

[2] T. Dubois, S. Jarrix, A. Penarier, P. Nouvel, D. Gasquet, L. Chusseau, B. Azaïs, "Near-Field Electromagnetic Characterization and Perturbation of Logic Circuits", IEEE Transactions on Instrumentation and Measurement, vol. 57, no 11, November 2008.

[3] A. Boyer, "Méthode de Prédiction de la Compatibilité Electromagnétique des Systèmes en Boîtier", Thesis, Université de Toulouse, October 2007.

[4] B. Vrignon, N. Lacrampe, F. Caignet, "Investigation of Effects of an ESD Pulse Injected by a Near-Field Probe into an Oscillator Block of a 16-Bit Microcontroller", EMC Compo 2007, 6th International Workshop on Electromagnetic Compatibility of Integrated Circuits, November 2007.

[5] A. Boyer, S. Bendhia, E. Sicard, « Modelling of a Mixed-Signal Processor Susceptibility to Near-Field Aggression », IEEE Symposium on EMC, Hawaii, July 2007.

[6] T. Dubois, S. Jarrix, J. Raoult, A. Penarier, P. Nouvel, B. Azais, D. Gasquet, "Electromagnetic susceptibility studies of op. amp. and a vco for a pll application," EMC Compo 2009, 7th Workshop International November 2009.

[7] T. Dubois, J. J. Laurin, J. Raoult, S. Jarrix, "On the Effect of Amplitude modulated EMI injected on a PLL Active Filter", EMC Compo 2011, 8th Workshop on Electromagnetic Compatibility of Integrated Circuits, November 2011.

[8] FEKO V14.0, EM Software, www.feko.info

[9] CST studio suite 2016, EM software, https://www.cst.com. 